\title{
Development and Pilot Evaluation of a Cancer-Focused Summer Research Education Program for Navajo Undergraduate Students
}

\author{
Edward R. Garrison, \\ Diné College, Shiprock, NM, USA \\ Mark C. Bauer, \\ Diné College, Shiprock, NM, USA \\ Brenda L. Hosley, \\ Arizona State University, Phoenix, AZ, USA
}

Christi A. Patten, Mayo Clinic College of Medicine, Rochester, MN, USA; Mayo Clinic Rochester, 200 First St. SW, Charlton 6-273, Rochester, MN 55905, USA

Christine A. Hughes, Mayo Clinic College of Medicine, Rochester, MN, USA

Mary A. Trapp, Mayo Clinic College of Medicine, Rochester, MN, USA

Wesley O. Petersen, Mayo Clinic College of Medicine, Rochester, MN, USA

Martha A. Austin-Garrison, Diné College, Shiprock, NM, USA

Clarissa N. Bowman, and

Diné College, Shiprock, NM, USA

\section{Robert A. Vierkant}

Mayo Clinic College of Medicine, Rochester, MN, USA

\section{Abstract}

This paper describes the development and pilot testing of a 10-week cancer research education program for Navajo undergraduate students. The program was piloted at Diné College with 22 undergraduates (7 men, 15 women) in 2007 and 2008. Students completed a pre-post program survey assessing attitudes, opinions, and knowledge about research and about cancer. The program was found to be culturally acceptable and resulted in statistically significant changes in some of the attitudes and opinions about research and cancer. Combining all 13 knowledge items, there was a significant $(p=0.002)$ change in the mean total correct percent from baseline [70.3 $(\mathrm{SD}=15.9)]$ to post-program [82.1 $(\mathrm{SD}=13.1)]$. The curriculum was adapted for a new cancer prevention and control course now offered at Diné College, enhancing sustainability. Ultimately, these efforts may serve to build capacity in communities by developing a cadre of future Native American scientists to develop and implement cancer research. 


\section{Keywords}

Native American; Cancer education; Cancer prevention

\section{Introduction}

The reduction of cancer health disparities is a major public health priority in the USA [1,2]. Cancer is the second leading cause of death among American Indian and Alaska Native (AI/ AN) people [3]. The New Mexico Cancer Registry ( 200,000 cases from 1969 to 2004) indicates that 14,130 cancers $(7 \%)$ occurred in $\mathrm{AI}$ in that state. Of these, 5,509 were Navajo, and the vast majority were living in the Navajo Nation at time of diagnosis. Kunitz [4] analyzed data from the Navajo Area Indian Health Service for New Mexico and Arizona and found that the crude average annual death rates from cancer increased significantly over the periods from 1972-1978 to 1998-2000 from 38.0 to 68.4 per 100,000. The 5-year relative survival for all cancer sites combined for AI/ANs is $35.2 \%$, which is the poorest survival from cancer among all population groups [5,6].

One reason for the high mortality is that Native Americans are more likely to be diagnosed with cancer at an advanced stage [1,6,7]. This often is attributed to lack of knowledge and misunderstandings about cancer, contrasting cultural beliefs, inadequate availability of services, and underutilization of services and treatment options that are available [8-10]. Other issues contributing to inadequate cancer prevention and treatment among Native American communities include lack of involvement of tribes in research study design and dissemination of research data and lack of targeted programs to strengthen the science education of $\mathrm{AI} / \mathrm{AN}$ youth and to promote mentored experiences in biomedical and public health research [11].

Nationally, few Native Americans complete Bachelor's degrees and only a small number go on to graduate school or professional programs [12]. From 2005 to 2006, AI/AN students accounted for $<1 \%(0.7 \%)$ of the total 1.5 million Bachelor's degrees awarded [12]. They represented only $0.3 \%$ of students entering medical school, a rate of matriculation far below those of other ethnic groups with the exception of Native Hawaiians and Pacific Islanders (American Association of Medical Colleges) [13]. Critical to increasing the number of Navajo Indian students entering biomedical fields is an enhanced understanding of the factors that influence retention $[14,15]$. Obstacles to pursuit of science degrees include the lack of role models and mentors in the sciences and in their communities, limited opportunities to obtain experience in a scientific field of study (i.e., through a summer internship), and cultural constraints such as support and encouragement from their families and communities to pursue a career in science [16-19]. Due to the paucity of those who complete science degrees, Native Americans serving as principal investigators in community-based cancer research projects are rare. However, AI/AN scientists' cultural perspectives are essential to the successful conduct of research to effectively address the burden of cancer in their own communities.

In 2006, Diné College and the Mayo Clinic Cancer Center (MCCC) received funding for a 4-year P20 planning grant from the Minority Institution/Cancer Center Partnership (MI/ $\mathrm{CCP}$ ) program of the Center to Reduce Cancer Health Disparities of the National Cancer Institute. The goal of the partnership is to increase first and second year Navajo undergraduates' interest in and commitment to biomedical coursework and careers, especially in cancer research, that will benefit their community. The project has three aims focused on: faculty cancer research education, student cancer research education, and 
student training in cancer research. The current report focuses on one of these aims: student cancer research education.

Diné College was established by an Act of the US Congress in 1968 as the first Native American tribally controlled college. It is chartered by the Navajo Nation and operates eight campuses to serve a reservation community spread over 27,000 square miles. Diné College, a 2-year undergraduate institution, serves approximately 2,000 students. In 2004, Diné College became the first tribal college to establish a degree program in Public Health.

The MCCC is one of 61 medical centers designated by the National Cancer Institute as a comprehensive cancer center. Mayo Clinic has experience working with and serving AI/AN communities through its two Native American programs (Native CIRCLE and Spirit of EAGLES) and the former Native WEB. Respectively, these programs have disseminated culturally appropriate cancer and nutritional information to AI/AN communities, provided infrastructure and capacity building programs in cancer care and research to AI/AN communities, and trained Tribal/IHS nurses and Community Health Representatives to provide breast/cervix cancer screening training and education [20]. These programs have been directed by Judith S. Kaur, MD, the second of only two Native American medical oncologists in the USA [21].

MCCC's assets combined with Diné College's Native American student and faculty resources and history of community activity placed the two institutions in an exceptional position to effectively reach the Navajo community. This paper describes the development and pilot testing of a cancer research education program for Diné College undergraduate students.

\section{Methods}

\section{Diné Educational Philosophy}

In the development and design of this new program for students, we drew from the Diné Educational Philosophy (DEP) which informs and guides college-wide curriculum development and reform including public health education [22]. Developed by Navajo cultural specialists, this philosophy represents the essence of the Navajo outlook on life. The core philosophical concept around which the DEP is based is the statement Sa'qh Naaghái Bik'eh Hózhóón, defined as "The Diné traditional living system, which places human life in harmony with the natural world and the universe. The philosophy provides principles both for protection from the imperfections in life and for the development of well-being" (General Catalog of Diné College). There are four key words, each associated with a multitude of symbolic and philosophical values, which provide a practical framework around which all aspects of college life and function, including curriculum, are developed. These are: 
Nitsáhákees

(Thinking)

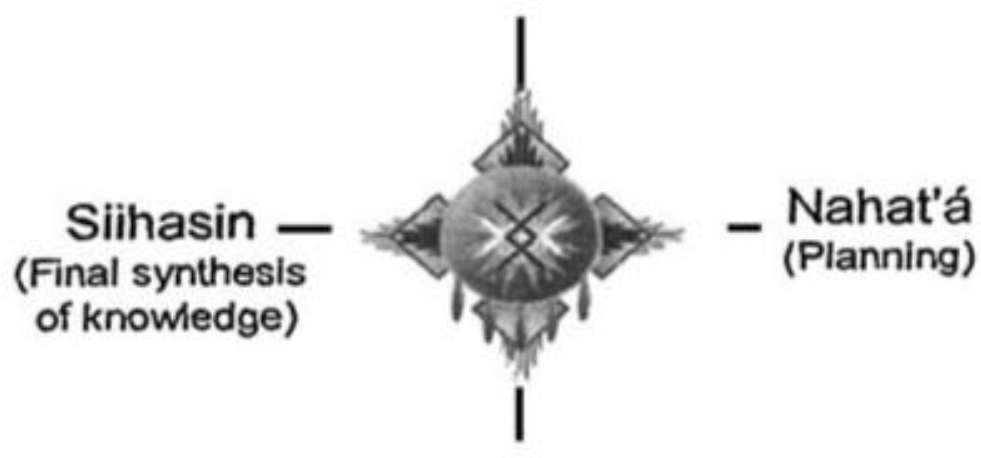

liná

(Living-implementing)

Nitsáhákees—thinking, assessing, reasoning

Nahat'á - planning, action, putting ideas into place

Iiná-achievement of outcomes, products, solutions, goals

Sii hasin-demonstrate competency, confidence

Summer Research Enhancement Program-Summer Research Enhancement Program (SREP) is a 10-week summer enrichment training program in public health research methods for Native American undergraduate students. The program introduces students to the research process and to health professions, providing a foundation for assisting in basic public health research and evaluation projects. SREP students bring with them their own cultural and traditional experiences, values and knowledge, and the program attempts to build upon these unique experiences from a broader perspective. This is accomplished by integrating the concepts of holism, balance/harmony, kinship, and relatedness shared among AI/AN people. These broader perspectives assist students in drawing their own conclusions as to how they may play a role in organizing and conducting research in their own communities.

SREP was originally designed for students with an interest in diabetes prevention research as a component of the National Diabetes Prevention Center that was established in Gallup, New Mexico. Students participating in the program earn seven transferable college credits (a four-credit Public Health Research Methods course and a three-credit Research Practicum course). They receive a $\$ 3,200$ stipend, housing in the campus dormitories for the 4 weeks of the program when they are in residence at Diné College, and three meals per day in the college cafeteria during those 4 weeks. For their 6-week practicum, they reside in their home communities and work in a local program or agency. The 10-week program consists of three stages as follows:

Stage 1: This stage involves 3 weeks of intensive training at the Diné College campus at Tsaile, Arizona where the students develop skills in public health research techniques. Students participate in education on public health, epidemiology, statistics, health promotion and disease prevention, cancer and diabetes disease processes, and research methods topics. The students develop competence working together in small groups on a hypothetical group public health intervention project in a designated Navajo community and then present their 
proposed interventions to their classmates and Diné College faculty and visitors at the conclusion of the first 3 weeks.

Stage 2: This stage involves a 6-week mentored research practicum experience. Each student is placed at a location in or close to his or her own community, in a cancer or diabetes prevention research or services program, where they contribute to a variety of ongoing activities. Placements are at programs on and near the Navajo Nation, primarily with the Navajo Area Indian Health Service or the Navajo Nation Division of Health. The practicum is designed to empower the student with "real-time" practical knowledge and research skills, including data collection, for community-based research projects.

The core objective of the practicum is to assist the student in the application to a current real-life program of data collection and analytic methods and techniques utilizing concepts of public health and health promotion and disease prevention and incorporating culturally relevant aspects of disease prevention where appropriate. The goal is for the student to be able to apply his or her newly acquired knowledge of experimental design and statistical analysis in either a quantitative and/or qualitative research setting or in a health agency services setting. The student is encouraged to build upon this knowledge and adopt a strong bicultural approach in the research activities, bridging Native American healing knowledge and Western biomedical knowledge. Active participation with program personnel is an integral part of the practicum expectation.

SREP program objectives address outcomes both for the students and for the program in which they have been placed for their practicum. The students are expected to gain practical, hands-on experience engaging with professional staff and community members in health promotion, clinical, or research activities, participation in planning and conceptualizing health programming, and doing their own work with actual data collected through health activities or research. Objectives for the impact that the program should have on the practicum site and for the benefit of the mentor at that site include expectations that the student should directly contribute to the accomplishment of the ongoing objectives of the site, providing extra personnel resources to serve more clients or conduct more activities. The data analysis that the student completes as part of the SREP practicum placement should be designed to provide the site with useful data summaries and some tested relationships that could inform further development of improved approaches or confirm positive results of the established services. Experience has shown that having a SREP student at a community health agency site helps to stimulate a discussion of the kinds of data available at the site and its potential use to inform the program. We have repeatedly found that program personnel are so fully occupied with day-to-day demands that they seldom have the time (or, in some cases, the training) to review program service data or to consider how that data might enhance program evaluation. In discussions between the student, the practicum mentor, and the SREP staff, evidence-based approaches drawing on available data for that site can be brought up and considered, and throughout the student's practicum placement, such issues are highlighted.

Stage 3: During the final stage, the students return to the Tsaile campus of Diné College and participate in a 1-week session where their practicum experiences are shared. Each student analyzes the data gathered during the practicum and prepares a formal, individually delivered presentation of the research service that he or she provided for the practicum site. The presentation consists of a description of the placement site, community setting, issues at that site, a research question and hypothesis, needs statement and literature review, methods, analysis of data, results and conclusion, and discussion. 
Cultural activities-In addition to the public health coursework and the practicum experience, the students participate in a variety of cultural activities during the program. The intent is to reinforce the students' understanding of the conceptual basis for more fully appreciating Native American lifestyles, emphasizing their relevance to contemporary life. Students are more likely to develop an interest in prevention research if those interests are nurtured in a culturally supportive environment. In the first morning session of the 10-week program, SREP is initiated with a blessing and welcome in a traditional ceremonial hooghan, conducted by the President of Diné College or other leadership at Diné College. A hooghan is a round building, set in a specially chosen place, which becomes a center of strength, clear thinking, and sound planning, and is used for Navajo healing ceremonies. The following evening, there is a talking circle. Talking circles, a group-based format, are a Native American cultural tradition where people gather together in a circle and each person shares his/her personal story or thoughts related to a particular topic. The students also participate in various physical activity sessions including a team building Ropes Course in Shiprock, NM, a Navajo communityled "Just Move It" fun walk/run, and a walk through Canyon De Chelley to White House Ruin (an ancient Native American cliff dwelling), and collectively gain experience in the use of a pedometer to monitor daily activity.

The program has had nine successful years of operation and averages about 12 students each summer. Between the years 2000 and 2006, 90 students participated, representing 21 tribes from across the USA. In 2007 and 2008, the program was expanded to include a cancer focus as one of the many components of the P20 collaborative project between Diné College and Mayo Clinic.

\section{Development of Cancer SREP}

Through the P20 grant collaboration, we planned, developed, piloted, and refined a Cancer SREP at Diné College. Information on cancer research was first introduced to the 2005 SREP students. Mayo Clinic faculty provided presentations based on their experiences with respect to community-based participatory cancer research topics including tobacco cessation (CAP) and colorectal cancer screening and prevention (MAT). Community outreach activities were also shared with the students, and community educational resources relevant to cancer were distributed for their use. The upcoming implementation of the P20 project was also discussed with the students and faculty in the format of a focus group, including the vision for a cancer-focused SREP, and feedback was obtained. This process was repeated at the 2006 SREP.

In the Spring of 2007, several members of the Mayo Clinic and Diné College team met for a 3-day working retreat to develop a cancer prevention, education, and research focus for SREP. The team used various resources available including Weinberg's The Biology of Cancer [23] and Web sites including those of the National Cancer Institute. Feedback on the curriculum for Cancer SREP was obtained from our national advisory committee formed for the P20 project comprising Native American scientists as well as others conducting cancer research within Native American communities. In addition, the curriculum was reviewed by a community advisory committee comprising Navajo health care providers and other community members.

The general structure of Cancer SREP followed that of previous SREPs, with three stages and cultural activities and diabetes topics included, but with a cancer focus added. During stage 1, on the first morning, the students were given a general overview of the diabetes and cancer tracks. After they had an opportunity to discuss the different program options and ask any questions they might have, the students made their decisions to focus on cancer or diabetes research (students had previously indicated their tentative selections during the application process). All students, regardless of track, participated in education on public 
health, epidemiology, statistics, health promotion and disease prevention, relevant disease processes, and research methods topics and developed a group presentation as described above. During stage 2, each student was placed in a diabetes or cancer prevention research program.

Table 1 shows the general topics, cancer topics, and diabetes topics covered in both the 2007 and 2008 programs. The diabetes topics followed the basic structure of SREP in previous years. As indicated in the table, there were 29 general topics provided to all students as a whole regardless of their chosen track, which included both diabetes and cancer topics. Five of the seven diabetes topics were provided only to students within the diabetes track, while two were presented to all students. Three of the nine cancer topics were presented only to students within the cancer track, while six were provided to all students. In addition to presentations, Mayo Clinic faculty participated by helping the students with their daily homework assignments and providing assistance and feedback on their group projects. In addition, there were several visiting faculty members from other institutions who presented and assisted the students.

Based on the feedback obtained from students at the end of the 2007 program, there were very few minor changes made to the 2008 program. Specifically, in 2008, there was a more in-depth focus on nutrition and other risk factors provided to all students. In addition, all students watched the cancer video Healthy Navajo Women: Walk in Beauty followed by a group discussion. Because the topics were nearly identical in both years and because of the small sample size for each pilot evaluation ( $\mathrm{N}=11$ ), we combined the 2007 and 2008 student groups for evaluation of the quantitative items on the pre-post test surveys.

\section{Pilot Evaluation}

Cancer SREP was piloted in the summers of 2007 and 2008.

Students-In 2007, the class consisted of three men and eight women, with an average age of 22 (range 19-26). In 2008, the class consisted of four men and seven women, with an average age of 32 (range 19-59). Four of the students in 2007 and six enrolled in 2008 chose a cancer focus for their summer research.

Measures-All students completed a pre- and post-program survey assessing attitudes, opinions, and knowledge about research and about cancer. Baseline surveys were administered on the first morning of the program. The items are shown in Tables 2 and 3. Responses assessing students' opinions and attitudes were collected using four-point Likert scale variables with possible responses of "strongly agree," "agree," "disagree," and "strongly disagree." Response options for the knowledge questions were "true," "false," and "I don't know." The survey also asked for qualitative feedback on whether the student would recommend the program, what was the most important thing he/she had learned, and how the student would use the knowledge and skills gained from their participation in the program.

Short-term outcomes of participation in SREP were assessed in annual surveys conducted by email: retention in educational program, completion of Associate of Science then Bachelor of Science degrees, retention in major (in this case, in some kind of area related to SREP), additional research or other internship, or presentation after SREP, and employment in fields using SREP skill/knowledge set. 


\section{Statistical Methods}

Integer values from 1 to 4 were assigned to the four response options in the Likert survey questions. Because some of the questions were positively phrased (such that strongly agree was the most appropriate response) and others were negatively phrased (such that strongly disagree was the most appropriate response), variable values were assigned such that higher values always corresponded to the more appropriate response. Questions assessing students' knowledge of cancer and research were re-coded as "correct" or "incorrect." Students responding "don't know" to any given question were grouped in the incorrect category. Data were summarized using means and standard deviations for ordinal variables and frequencies and percents for categorical variables. We examined pre-post program changes in the ordinally scaled opinion and attitude variables using paired $t$ tests. For each of the 13 knowledge questions, we compared the proportion of correct responses at baseline to the proportion correct post-program using McNemar's test of marginal homogeneity. Due to sparse table cells, exact tests (based on exact binomial probabilities) were used instead of the more traditional asymptotic chi-square tests. Next, we calculated the total percent of correct responses for a given student across all 13 questions, separately for the baseline and posttraining questionnaires. We then compared these baseline and post-training summary percentages using paired $t$ tests. Because our sample included only 22 individuals, we decided a priori to use one-tailed statistical tests to maximize power. For each variable, we tested for strict increases (in the ordinal values for the opinion and attitude questions and in the proportion of correct responses for the knowledge questions) from baseline to postprogram. All observed findings trending in the opposite direction of that hypothesized were deemed non-significant. Analyses were carried out using the SAS system (SAS Institute, Inc., Cary, NC).

\section{Results}

Table 2 shows pre-post program mean responses for the opinion and attitude survey items. Significant changes were observed for the items "Program evaluation is needed in my community," "I know how to conduct good research," and "I can make a difference in the health of my community" with an increase in the mean for these items pre-post. Table 3 shows the percent correct for the knowledge items at both time points. Combining all 13 knowledge items, the mean total correct percent at baseline was $70.3(\mathrm{SD}=15.9)$ and at postprogram was $82.1(\mathrm{SD}=13.1)$. The mean difference in total percent correct pre-post program was $11.9(\mathrm{SD}=16.6, p=0.002$, one-tailed paired $t$ test $)$.

The acceptability of the program in both years was high. All students indicated that they would recommend the program to another student. When the students were asked about the most important thing that they had learned, several themes emerged in the student responses. For example, students reported an increase in their knowledge and understanding of: (1) how to design and execute a research study, (2) how to utilize statistical methods for analysis and for presentations, and (3) the need for cancer research specifically on the Navajo Nation. For example, "I learned how to conduct cancer research and how to implement it in our community, in the future" and "I learned how to address a health topic...."When asked how they would use the knowledge and skills gained from their participation, the students reported that they would take the knowledge back to their community or family and use the information learned in future classes, research, and internships. Some specific comments were: "Participate more in community studies," "Get more information on diabetes and cancer for myself and my family members," and "Help out my community and make it a better place...." 


\section{Short-Term Student Outcomes}

For the 2007 and 2008 SREP students (N=22), $14(64 \%)$ are still in the educational pipeline, either with Diné College (five), another 2-year institution (two), or having transferred to a university (seven). That rate of retention compares favorably with the typical 1-year (44\%) and 2-year (35\%) retention rates of other Diné College students. Of those, 11 (79\%) remain in majors related to public health or health science, which is a fairly high rate. Six of the students are currently employed, with two employed in fields related to public health and the other four simply working for a period of time before returning to school. From the cohort of 22 students, eight have subsequently received Associate of Science degrees and one a Bachelor of Science degree. This rate (41\%) compares very favorably to a cumulative graduation rate of around 6\% after 3 years that is more typical of the Diné College student body. Four of these students went on to be selected for a higher level cancer research internship at Mayo Clinic, and three of them were able to take advantage of the opportunity and completed it successfully. Two other students went on to participate in other internships in science research areas. Four of the students participated in intensive multiple day training with the Native Investigator Research Training Program with the University of ColoradoDenver (sponsored by the Native Elder Research Center).

\section{Development of a "Cancer Prevention and Control" Course at Diné College}

The Cancer SREP pilot experience was used as the foundation for the development of a new three-credit course at Diné College entitled "Cancer Prevention and Control." The course was offered at Diné College for the first time during the Spring 2009 semester and included both biology and public health topics related to cancer, causes of cancer, and cancer prevention and control. Students had the option of enrolling in the course either as a Biology course or as a Public Health course. ${ }^{1}$ The course was offered via interactive television at six of the eight campuses of the Diné College system (two campuses in New Mexico and four campuses in Arizona), with a total of 22 students enrolled at all campuses.

\section{Discussion}

This project addressed a considerable gap in the field of cancer education. Some programs have been developed to stimulate interest in a research career among Native Americans through masters, doctoral, or post-doctoral level training [18,24]. Other programs have been designed to enhance Native American undergraduates' interests in pursuing a career in medicine [15]. These include the University of Minnesota, Native Americans into Medicine program (http://www.caimh.org) and the University of New Mexico Bridges Program (http://hsc.unm.edu/som/fcm/CNAH/index.shtml). However, these programs have not focused on cancer research education specifically. It is important to reach Navajos early in their post-secondary education to enhance their skills in conducting research and interest in pursuing science degrees. In this project, we developed a cancer research education program (Cancer SREP) for Navajo and other AI/AN undergraduates. The program was found to be culturally acceptable and resulted in changes in some of the attitudes and opinions about research and about cancer. Short-term student outcomes following the SREP program were also favorable. Moreover, the curriculum was adapted for a new "Cancer Prevention and Control" course that is now offered as an elective at Diné College, enhancing sustainability. Ultimately, these efforts may serve to build capacity in Native American communities by developing a cadre of future scientists to develop and implement cancer research that will benefit the health of AI/AN people [21,25].

\footnotetext{
${ }^{1}$ A copy of the syllabus for the "Cancer Prevention and Control" Public Health course at Diné College is available upon request from the first author.
} 
A limitation is the small sample which likely limited our statistical power to detect significant changes on other attitude/opinion findings. We also found that knowledge about cancer increased significantly pre-post program based on the total percent correct for the 13 knowledge items. We did not analyze the data separately for those who chose a cancer or diabetes track, respectively, because of the small sample size within each subgroup and because all students were exposed to cancer and diabetes topics. The items included were very general with respect to cancer knowledge given that the course was an introduction to cancer prevention and control. Future evaluations could include more specific cancer knowledge items and inclusion of case studies as part of the assessment. This would allow for testing of assimilation and application of knowledge.

With respect to "lessons learned," through the process of developing and implementing the curriculum, we have learned that efforts are needed to improve the recruitment of Cancer SREP students. There is a need to develop different cultural approaches to engage the community in a dialogue about cancer prevention and research which would help to increase family and community support for student participation in such activities as SREP. The new "Cancer Prevention and Control" course now being offered at Diné College is also a potential avenue to reach and engage potential students to participate in Cancer SREP. Moreover, we learned that there is a need to work with potential mentors to increase the perceived value of mentoring students and also to be creative in finding placements that address health promotion improving the number of possible mentors for the students. Moreover, prospective follow-up of students who participated will be needed to assess longterm outcomes such as completion of a graduate degree.

\section{Acknowledgments}

This project was supported by grants P20 CA118774 and P20 CA119013 from the MI/CCP program of the National Cancer Institute. Support was also provided by grant 5 R25 GM061396 at Diné College from the Research Initiative for Scientific Enhancement (RISE) program of the Minority Biomedical Research Support (MBRS) program of the National Institute of General Medical Sciences. The authors appreciate the feedback and guidance on the development of Cancer SREP from our P20 project national advisory committee members: Dr. Judith Kaur and Dr. Teri Britt Pipe, Mayo Clinic; Dr. Jennie Joe, University of Arizona; Dr. Charles Wiggins, University of New Mexico; Dr. David Burgess, Boston College; Amanda McNeill and Jack C. Jackson, Sr., Diné College. We would also like to thank Frank Morgan, Cultural Consultant; Dr. Ferlin Clark, President of Diné College; and members of our local community advisory committee for their feedback and support of this project. In addition to the authors, we acknowledge the faculty who presented at Cancer SREP from (1) Diné College-Ferlin Clark, Ph.D., President; Jack C. Jackson, Sr., M.A.; Victoria Beatty, M.L.S.; Verena J. Serafin, M.P.H.: (2) University of Arizona, College of Public Health—Carol Goldtooth-Begay, M.P.H.; Nicolette Teufel-Shone, Ph.D.: (3) University of New Mexico-Shirley Srouji, M.S.: (4) Gallup Indiana Health Service-Bernadine Toya, M.S.N.; (5) University of Colorado Health Science Center-Spero M. Manson, Ph.D.: (6) Navajo Nation Breast and Cervical Cancer Prevention Program-Janice Jumbo, M.P.H.: (7) Chinle Service Unit, Navajo Area IHS—Johnson Dennison, M.A.; Gerald King, B.S.: (8) Navajo Division of Health: Kayenta Public Health Nursing Program-Linda Cothron, R.N., M.S., A.R.N.P., O.C.N.; Dennis Whiterock, Cancer Survivor; (9) Cultural Consultant—Frank Morgan, B.S.

\section{References}

1. Espey DK, Wu XC, Swan J, Wiggins C, Jim M, Ward E, et al. Annual report to the nation on the status of cancer, 1975-2004, featuring cancer in American Indians and Alaska Natives. Cancer 2007;110(10):2119-2152. [PubMed: 17939129]

2. US Department of Health and Human Services. Making cancer health disparities history. Report of the Trans-HHS Cancer Health Disparities Review Group; Washington, DC: 2004.

3. Cobb N, Paisano RE. Patterns of cancer mortality among Native Americans. Cancer 1998;83(11): 2377-2383. [PubMed: 9840538]

4. Kunitz SJ. Ethics in public health research: changing patterns of mortality among American Indians. Am J Public Health 2008;98(3):404-411. [PubMed: 18235064] 
5. Ries, LA.; Young, JL.; Keel, GE.; Eisner, MP.; Lin, YD.; Horner, JJ. SEER survival monograph: cancer survival among adults. National Cancer Institute; Bethesda, MD: 2007. U.S. SEER Program, 1988-2001. Patients and tumor characteristics. NIH Pub No. 07-621556. SEER Program

6. Clegg LX, Li FP, Hankey BF, Chu K, Edwards BK. Cancer survival among US whites and minorities: a SEER (Surveillance, Epidemiology, and End Results) Program population-based study. Arch Intern Med 2002;162(17):1985-1993. [PubMed: 12230422]

7. Li CI, Malone KE, Daling JR. Differences in breast cancer stage, treatment, and survival by race and ethnicity. Arch Intern Med 2003;163(1):49-56. [PubMed: 12523916]

8. Burhansstipanov L, Tenney M, Russell S, Bad Wound D, Plomer K. Overview of the Native American Cancer Conference III. Risk factors, outreach and intervention strategies. Cancer 1996;78(7 Suppl):1527-1532. [PubMed: 8839565]

9. Swan J, Edwards BK. Cancer rates among American Indians and Alaska Natives: is there a national perspective. Cancer 2003;98(6):1262-1272. [PubMed: 12973851]

10. Wilson RT, Adams-Cameron M, Burhansstipanov L, Roubidoux MA, Cobb N, Lynch CF, et al. Disparities in breast cancer treatment among American Indian. Hispanic and non-Hispanic White women enrolled in Medicare. J Health Care Poor Underserved 2007;18(3):648-664. [PubMed: 17675720]

11. US Department of Health and Human Services. Facing cancer in Indian country. President's Cancer Panel 2002; Washington, DC: 2002.

12. NCES (National Center for Education Statistics). Bachelor's degrees conferred by degree-granting institutions by race/ethnicity and sex of student, 2005-2006. 2008. Table 274

13. AAMC (Association of American Medical Colleges). Diversity in medical education: Facts \& Figures 2008. 2008. p. 33Figure 15

14. Cora-Bramble D. Minority faculty recruitment, retention and advancement: applications of a resilience-based theoretical framework. J Health Care Poor Underserved 2006;17(2):251-255. [PubMed: 16702712]

15. Sequist TD. Paving the way-providing opportunities for Native American students. N Engl J Med 2005;353(18):1884-1886. [PubMed: 16267318]

16. Brown LL, Kurpius R, Sharon E. Psychosocial factors influencing academic persistence of American Indian college students. J Coll Student Dev 1997;38(1):3-12.

17. Harris DL, Mullan F, Simpson CE Jr, Harmon RG. The current and future need for minority medical faculty. J Assoc Acad Minor Phys 1991;2(1):14-17. [PubMed: 1811814]

18. Henly SJ, Struthers R, Dahlen BK, Ide B, Patchell B, Holtzclaw BJ. Research careers for American Indian/Alaska Native nurses: pathway to elimination of health disparities. Am J Public Health 2006;96(4):606-611. [PubMed: 16507731]

19. Jackson AP, Smith SA. Postsecondary transitions among Navajo Indians. J Am Indiana Education 2001;40(2):28-47.

20. Kaur JS, Dignan M, Burhansstipanov L, Baukol P, Claus C. The "Spirit of Eagles" legacy. Cancer 2006;107(8 Suppl):1987-1994. [PubMed: 16944468]

21. Kaur JS, Hampton JW. Cancer in American Indian and Alaska Native populations continues to threaten an aging population: the need for tribal, state, and federal action. Cancer 2008;113(5 Suppl):1117-1119. [PubMed: 18720371]

22. Garrison ER. The Diné Educational Philosophy (DEP) and its incorporation into the Associate of Science Degree Program in Public Health at Diné College. J Interprof Care 2007;21:64-78. [PubMed: 17896247]

23. Weinberg, R. Garland Science. Taylor \& Francis Group; New York: 2006. The biology of cancer.

24. Baquet CR, Mack KM, Mishra SI, Bramble J, Deshields M, Datcher D, et al. Maryland's Special Populations Network. A model for cancer disparities research, education, and training. Cancer 2006;107(8 Suppl):2061-2070. [PubMed: 16944470]

25. Clark NM, McLeroy KR. Creating capacity through health education: what we know and what we don't. Health Educ Q 1995;22(3):273-289. [PubMed: 7591785] 
Table 1

Cancer SREP curriculum 2007 and 2008

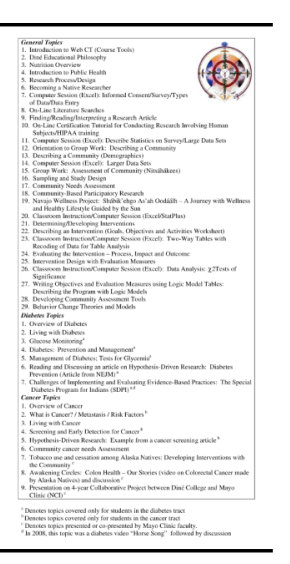




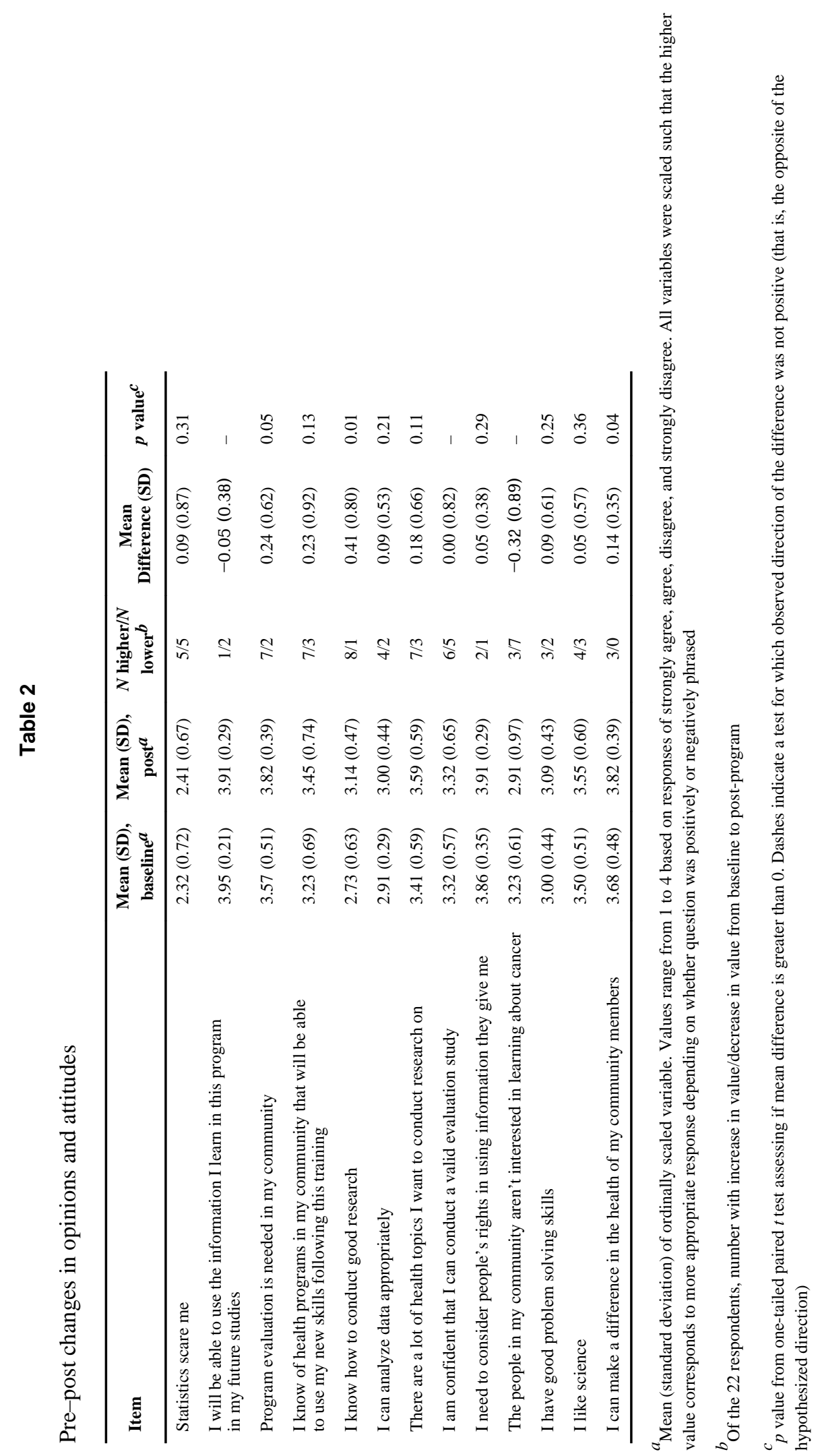




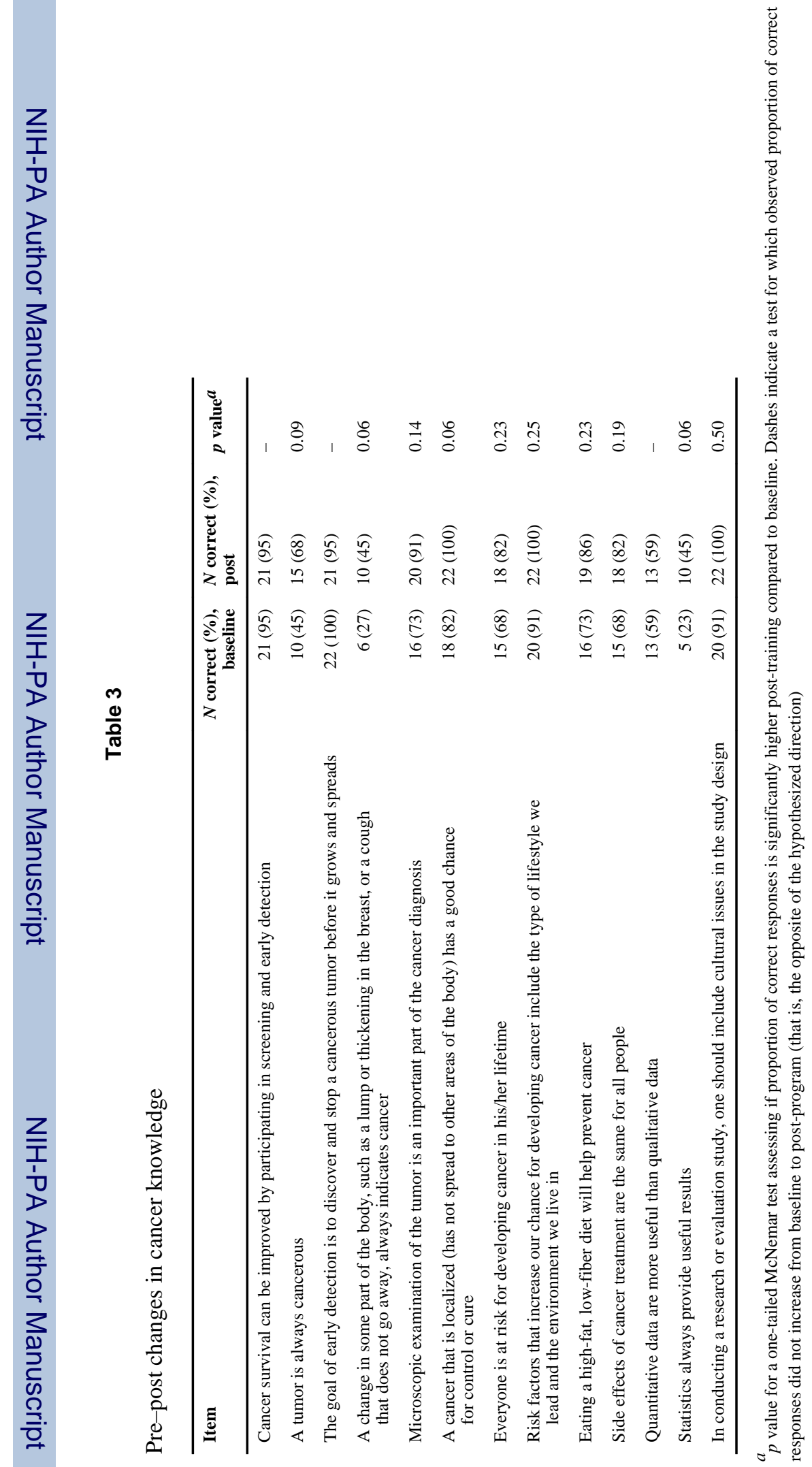

